\title{
A Multidisciplinary Approach in Management of Breast Cancer: Case Study and Literature Reviews
}

\author{
Andee Dzulkarnaen Zakaria*, Nur Farhana Abd Salam, Wan Zainira Wan Zain, \\ Mohd Nizam Hashim
}

School of Medical Sciences, Universiti Sains Malaysia, Kubang Kerian, Kelantan, Malaysia.

Email: *andee@kb.usm.my

Received May $14^{\text {th }}, 2013$; revised June $16^{\text {th }}, 2013$; accepted June $24^{\text {th }}, 2013$

Copyright (C 2013 Andee Dzulkarnaen Zakaria et al. This is an open access article distributed under the Creative Commons Attribution License, which permits unrestricted use, distribution, and reproduction in any medium, provided the original work is properly cited.

\begin{abstract}
The diagnosis of breast cancer can cause a significant anxiety in someone's life. The care of the breast cancer patient requires in many centres. The care of breast cancer patient is undertaken as a joint venture between the surgeon, medical oncologist, radiotherapist, pathologist and allied health professionals such as the clinical nurse specialist as well as counsellors. This is a case of a 39-years-old Malay woman presented to HUSM with a history of ulcerating breast lump with bloody pus discharge on the right breast. An initial mammogram study showed lesion suspicious of malignancy, and histopathological examination was consistent with invasive ductal carcinoma. She received neoadjuvant chemotherapy before proceeding to right modified radical mastectomy with axillary clearance and immediate chest wall reconstruction. Detailed discussion on the diagnosis, treatment, surgical intervention and progress of the patient with reference to available literature reviews are presented.
\end{abstract}

Keywords: Breast Cancer; Multidisciplinary Approach; Reconstructive Surgery

\section{Introduction}

A 39-years-old single, nulliparous Malay woman, came to our institution with a complaint of right breast lump since one year duration. She has no family history of breast cancer and other malignancies such as ovarian, endometrial and colorectal cancer. Her past medical history is unremarkable. She attained menarche at the age of 12. She was initially presented with a lump located at the right upper outer quadrant, about the size of a peanut. It was described as a single lump, round in shape and soft in consistency. However, there were no complaints of tenderness, skin changes on the breast area or any nipple discharges. She only went to a tertiary centre as she felt that the breast lump is getting slightly bigger around 4 months after the initial presentation. Several investigations were done for her, including mammogram study (Figure 1). The initial analysis of the imaging study was graded according to the Breast Imaging Reporting and Data System (BIRADS) as BIRADS 4. The core biopsy revealed an infiltrating ductal carcinoma (Figure 2) with positive estrogen and progesterone (ER/PR). cERB was

"Corresponding author. found to be not overly-expressed.

The final diagnosis of breast cancer was revealed to

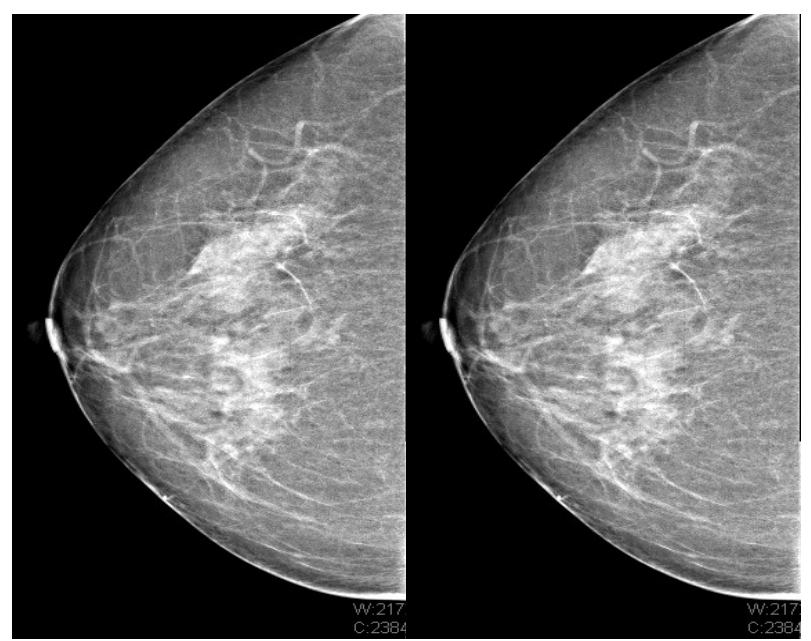

(a)

(b)

Figure 1. Mammogram in (a) medio-lateral oblique (MLO) and (b) cranio-caudal (CC) view showing lesions suspicious of malignancy located at the upper outer quadrant of the right breast. It was classified as BIRADS 4. 


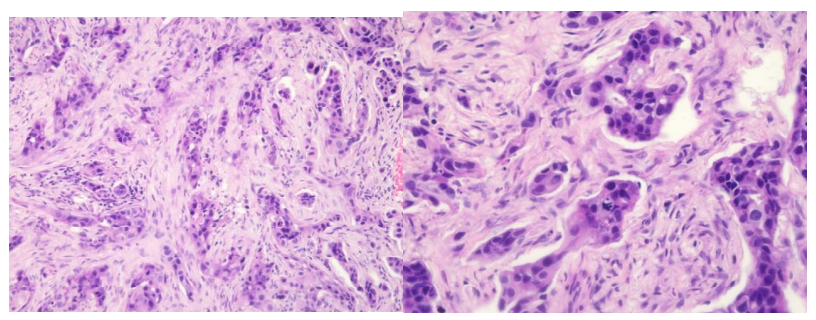

(a)

(b)

Figure 2. (a) Sections showed fibrocollagenous tissue infiltrated by malignant cells arranged mostly in cords and strands. Tubular formation is more than $75 \%$; (b) The cells display mild to moderate pleomorphism with vesicular or hyperchromatic nuclei. Mitoses are 7/10 in high power field. Both (a) and (b) showed features consistent with infiltrating ductal carcinoma.

her. She was given the option of surgery however she did not come for the follow up. Instead, she went to traditional healers; however, there was no history of manual manipulation or massage done over the lump area. The lump continued to grow progressively. About 3 months prior to current admission, she started to experience an intermittent throbbing pain over the lump area. Initially, she was able to tolerate the pain. However, the pain became progressively worse and started to affect her daily activity.

A week prior to admission, bloody pus discharges started to ooze out from the skin overlying the lump. The discharge was yellowish in colour with streak of blood. On top of that, the severity of the pain over the lump area was becoming unbearable, and she finally went to the emergency in our centre for further treatment.

\section{Physical Examination}

On examination, there was a $10.0 \times 8.0 \mathrm{~cm}$ fungating, ulcerating mass located mainly at the upper outer quadrant of the right breast (Figure 3). The shape was round and the margin is regular with "sloping edge". The floor of the ulcer is yellowish in colour with contact bleeding. Serous and bloody discharge was also seen and the discharge was foul smelling. Peau d' orange skin change was observed. The nipple-areolar complex was normal.

Lymph node examination revealed lymphadenopathy at anterior axillary line. The characteristic of the lymph node is single, round in shape, mobile, smooth surface, regular margin with firm consistency. The size is at $2 \times 3$ $\mathrm{cm}$.

The abdominal examination was normal with no organomegaly detected. Examination of other systems was normal.

\section{Investigations}

Several blood investigations were done for this patient including full blood count, blood urea and serum elec-

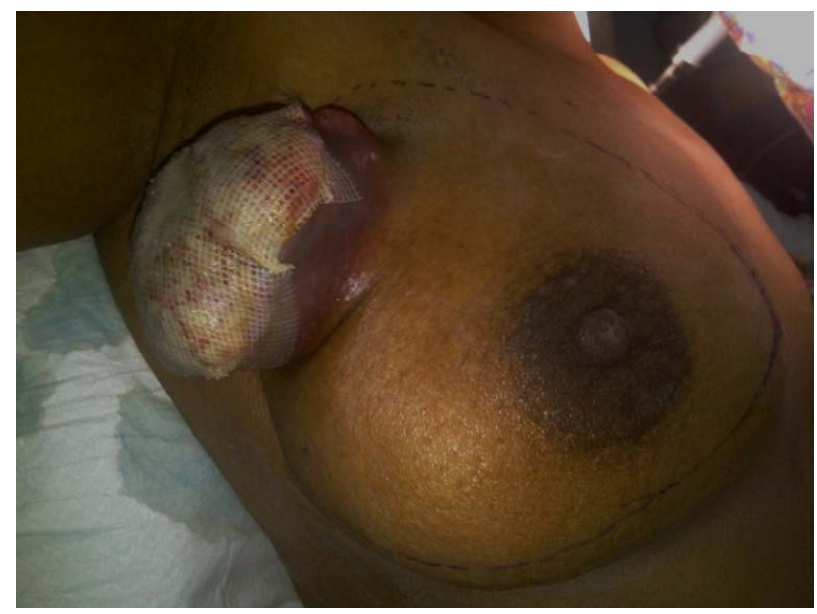

Figure 3. The fungating, ulcerous lesion is seen occupying parts of the upper quadrant of the right breast. Peau d' orange skin change can be observed. The nipple-areola complex is normal.

trolyte, serum calcium and liver function test. The level of white cell count, level of alkaline phosphatase (ALP) as well as serum calcium was found to be elevated, however other results were normal.

Blood and swab culture was also performed and there was no growth so far.

Imaging studies such as X-ray and CT scan were also done. The X-ray was normal with no evidence of metastasis to the lungs. CT scan was also normal with no evidence of distant metastasis to the vertebra and other organs such as the liver (Figure 4).

\section{Management}

Her hydration was maintained by administration of intravenous fluids. Intravenous antibiotic was also prescribed to cover infection in view of the raised white cell count. IV Unasyn (Ampicillin and Sulbactam) $1.2 \mathrm{mg}$ was given three times Fentanyl patch $12.5 \mathrm{mg}$ was also given every 72 hours to reduce the pain. Daily dressing was done with normal saline and Bactigrass dressing.

Patient was reviewed by the oncology team and neoadjuvant therapy was planned. A combination of taxanebased agent, adriamycin and anthracyclin dosage of 60 $\mathrm{mg} / \mathrm{m}^{2}$ (TAC) regimen was used. After 6 cycles of chemotherapy was completed, she underwent right modified radical mastectomy with axillary clearance and immediate chest wall reconstruction with unilateral pedicled transverse rectus abdominis muscle (TRAM) (Figure 5).

\section{Progress}

After the operation, her condition is stable and she is healing well. The skin flap over the defect area is viable and healing well with no complications noted so far such 


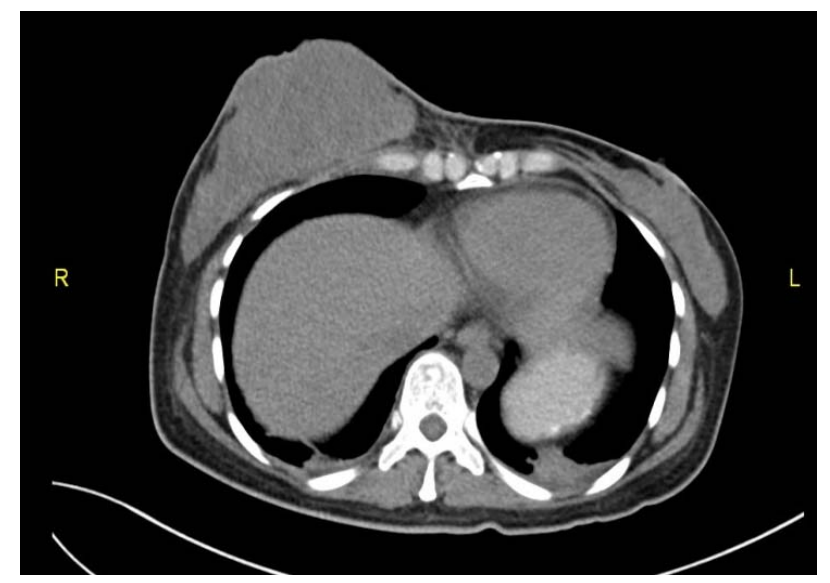

(a)

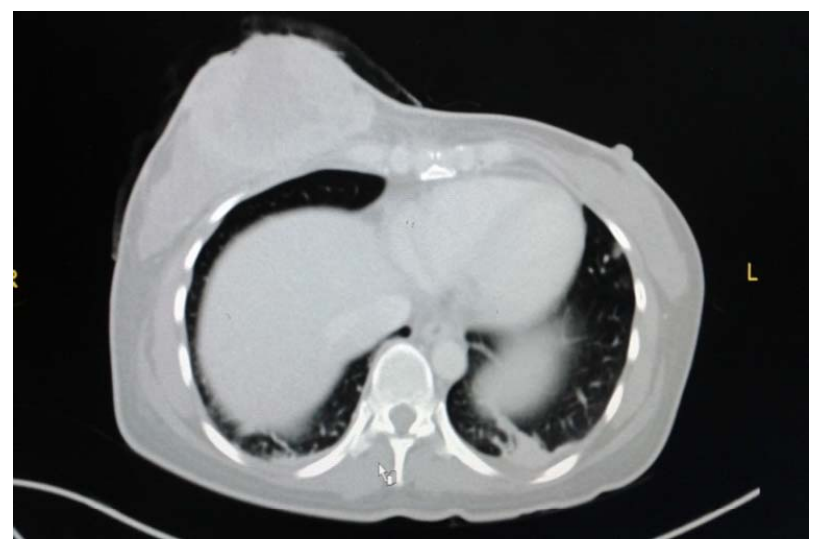

(b)

Figure 4. (a) Plain CT scan (b) Contrast-enhanced CT scan (CECT). Both showed no evidence of distant metastasis. The breast mass can be clearly demonstrated in both images.

as infection and wound dehiscence. Upon discharge, she is given appointment dates for follow up at our general surgery as well as the oncology clinic.

\section{Discussion}

A study by A. Jemal et al. (2008) revealed that breast cancer is the most common site-specific cancer in women and is the leading cause of death from cancer for women aged 20 - 59 years. It accounts for $26 \%$ of all newly diagnosed cancers in females and is responsible for $15 \%$ of the cancer related deaths in women $[1,2]$.

The risk factors for breast cancer may be divided into several categories such as demographic, estrogen exposure, lifestyle, personal breast characteristics, familial and inherited genetic mutations, and radiation exposure. Risk is generally grouped as minor and major. Minor risk factors increase a woman's lifetime risk from $12 \%$ to approximately $15 \%$. A woman's age is the strongest risk factor for breast cancer with $85 \%$ of women with breast cancer was noted to be more than 40 years old [2,3].

Katz et al. stressed again that approximately $15 \%$ to
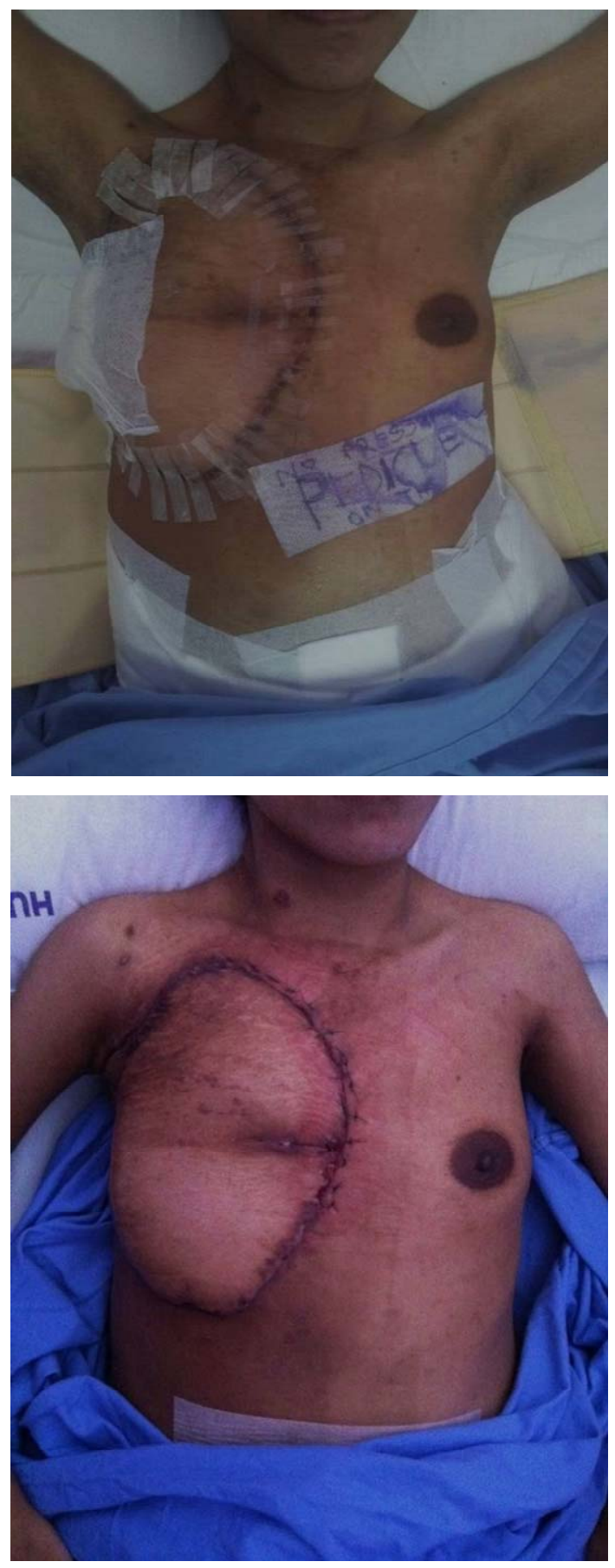

Figure 5. Patient few days after the operation. The flaps are healing well with no complications observed.

$20 \%$ of breast cancers are associated with a familial or inherited risk. (Thus, $80 \%$ to $85 \%$ of women have no significant familial risk factors.) By far the most common is the mutations in the BRCA 1 and 2 genes. Less common include the Li-Fraumeni syndrome, associated with the p53 gene, and Cowden syndrome, associated with the PTEN gene [3,4].

There have been numerous classifications of breast 
carcinoma including histopathology classification, gene profiling and receptors present and also clinical staging. Based on histological classification, infiltrating ductal carcinoma is the most common breast malignancy, accounting for $75 \%$ of cases. Approximately $10 \%$ of infiltrating ductal carcinomas are of a uniform histologic picture and are then classified as medullary, colloid, comedo, tubular, or papillary carcinomas $[3,4]$.

In order to reach a final diagnosis, the triple assessment method should be applied in any patient who presents with breast lump or other symptoms suspicious of carcinoma. The triple assessment consists of clinical assessment (history taking and physical examination), imaging studies as well as histopathology studies. This approach can increase the positive predictive value (PPV) to exceed $99.9 \%$ [5].

In terms of treatment of cancer of the breast, the basic principles are to reduce the chance of local recurrence and the risk of metastatic spread. Treatment of early stage of breast cancer will usually involve surgery with or without radiotherapy. In an event where there are adverse prognostic factors such as lymph node involvement that increase the likelihood of metastatic relapse, systemic therapy such as chemotherapy or hormone therapy is added. At the other end of spectrum, locally advanced or metastatic disease is usually treated by systemic therapy to palliate symptoms, with surgery playing a much smaller role as mentioned by Williams et al. [5].

Kaufmann, M. et al. stated that neoadjuvant chemotherapy (also called as primary systemic therapy or induction therapy) is standard management for women who have locally advanced or inflammatory breast cancer but can be applied to all women who may require postoperative chemotherapy for early-stage breast cancer. Disease-free survival and overall survival are equivalent between patients treated with neoadjuvant chemotherapy and patients treated with the same regimen postoperatively. There are various advantages that can be derived from this approach. Preoperative chemotherapy can offer women less morbid surgical treatment by down-staging both the primary breast tumor and axillary metastases. Finally, clinicians can be informed of the chemosensitivity of the tumor by looking at the response to chemotherapy, and can predict long-term outcome for women who have breast cancer [6].

Kaufmann, M. et al. also stated that in terms of the regimen of neoadjuvant, anthracycline-based regiment is the most extensively studied regimen. The typical approach consists of at least four to six cycles of an anthracycline-based regimen, usually adriamycin and cyclophosphamide, with or without the addition of taxane-based agents [6]. Several studies including by Bear, H.D., et al. in 2003 have reported higher pathologic clinical response rates and rates of BCS with the addition of preoperative docetaxel. Nonetheless, taxane and anthraxcycline regimens appear to be the most successful [7].

Mastectomy is indicated in for large tumors, multifocal disease, central tumors beneath or involving the nipple, local recurrence or patient's reference. The radical Haslted mastectomy is no longer indicated as it caused excessive morbidity with no survival benefit. The modified radical (Patey) mastectomy is more commonly performed. The excised mass is composed of the whole breast, a large portion of the skin, the centre which overlies the tumor but which always include the nipple, and all the fat, fascia and lymph nodes of the axillary [5].

Few studies look at the psychological and social adjustments to mastectomy in 160 women, who were followed at intervals of 3,12 , and 24 months after surgery. One in four women was reported to be in a state of depression and associated with marital and sexual problems 2 years after the initial therapy. The distress that comes from mastectomy may be more pronounced in younger women, as a result of significant treatment-related distress and changes in body image and sexuality during the course of the illness as observed by Fobair, P. et al. [8].

According to Nahabedian, M.Y., et al., patients under 50 years of age have a 4.3-fold greater likelihood of undergoing mastectomy followed by breast reconstruction than older patients [9]. The reasons behind this is described by Peppercorn, J., et al., which include the desire to achieve adequate breast cosmetic and symmetry, as well as fear of recurrence due to the fact that breast tumors in young women are more likely to be associated with high-risk tumor features and poorer prognosis, and tend to present with a more advanced stage at diagnosis [10].

Petit, J.Y., et al. showed that immediate breast reconstruction is now recognized as a safe procedure from the oncological point of view and is able to offer a series of advantages, such as better esthetic results compared with delayed reconstruction, attenuation of the sense of mutilation deriving from the mastectomy and a reduction in surgical times [11]. However, extra care must be taken in-patient who needs post-operative radiotherapy. Studies done by Kronovitz, S.J., et al. showed that there is two main problems exist for performing immediate reconstruction on patients who will then undergo RT. First, RT can undo the esthetic result from immediate reconstruction due to impairment of wound healing process and capsular contracture phenomena. Secondly, it can create problem especially when planning and designing the irradiation field. The slope of breast profile after reconstruction and the non-uniform thickness of the thoracic wall can cause under dosage radiation and non-homogenous of dose within the fields of radiation respectively [12].

Nahabedian MY et al. emphasized that in 30\% of cases, 
particularly following RT, reconstruction with autologous tissue is to be preferred [9]. The main myocutaneous flaps used in breast reconstruction are the transverse rectus abdominis myocutaneous (TRAM) flap introduced by Hartrampf in 1982 and used both as a pedicle flap or free (anastomosis between the inferior epigastric artery and the thoracodorsal artery, subscapular or internal breast), combined with or without prosthesis [9,13,14].

Pedicle TRAM flap uses abdominal muscle, fat and skin tissue vascularized by the rectus muscle pedicle to reconstruct the breast mound. Grossly, in the pedicle TRAM flap, excess skin, subcutaneous fat and rectus muscle from the infraumbilical area are transferred through a subcutaneous tunnel to the ipsilateral or contralateral mastectomy site. The flap is then rotated, shaped into a breast mound, and sutured; the umbilicus and the abdominal skin are sutured into its new position and the abdomen skin is sutured as in an abdominoplasty. Despite the loss of muscle function after a pedicle TRAM flap harvest, it is still possible for patients to become pregnant and carry a pregnancy to term, as well as to achieve a normal vaginal delivery [15].

Similar with our patient that in the free TRAM flap the skin, subcutaneous fat, deep inferior epigastric artery, and a small portion of the rectus muscle and fascia from the infraumbilical area are transferred to the chest defect, were epigastric vessels are reattached to either thoracodorsal or internal thoracic vessels via microsurgery. This technique allows the relocation of larger amounts of tissue with a lesser risk of fat necrosis. Hence, it may be a better procedure in patients with risk factors such as smoking, diabetes mellitus, and obesity [15].

\section{Conclusion}

Multidisciplinary approach is paramount and effective in breast cancer management. Despite the life changing diagnosis of breast cancer, reconstructive surgery opens an avenue of possibility to encourage surgery when needed, and improves the quality of life, physically and mentally.

\section{Consent}

Informed consent obtained from patient.

\section{REFERENCES}

[1] A. Jemal, R. Siegel, E. Ward, Y. Hao, J. Xu and T. Murray, “Cancer Statistics, 2008," A Cancer Journal for Clinicians, Vol. 58, No. 2, 2008, pp. 71-96. doi:10.3322/CA.2007.0010

[2] N. N. Baxter, B. A. Virnig, S. B. Durham, et al., "Trends in the Treatment of Ductal Carcinoma in Situ of the Breast," Journal of the National Cancer Institute, Vol. 96, No. 6, 2004, pp. 443-448. doi:10.1093/jnci/djh069

[3] V. L. Katz and D. Detters, "Lentz: Comprehensive Gy- necology Mosby an Imprint of Elsevier. Chapter 15. Diagnosis and Treatment of Benign and Malignant Breast Diseases,” 6th Edition, Elsevier, Philadelphia, 2012.

[4] S. J. Katz, P. M. Lantz, N. K. Janz, et al., "Patient InVolvement in Surgery Treatment Decisions for Breast Cancer,” Journal of Clinical Oncology, Vol. 23, No. 24, 2005, pp. 5526-5533. doi:10.1200/JCO.2005.06.217

[5] N. S. Williams, C. J. K. Bulstrode and P. R. O’Connell, "Bailey and Love's Short Practice of Surgery Textbook," 25th Edition, Edward Arnold, London, 2008.

[6] M. Kaufmann, G. N. Hortobagyi, A. Goldhirsch, et al., "Recommendations from an International Expert Panel on the Use of Neoadjuvant (Primary) Systemic Treatment of Operable Breast Cancer: An Update,” Journal of Clinical Oncology, Vol. 24, No. 12, 2006, pp. 1940-1949. doi:10.1200/JCO.2005.02.6187

[7] H. D. Bear, S. Anderson, A. Brown, et al., "The Effect on Tumor Response of Adding Sequential Preoperative Docetaxel to Preoperative Doxorubicin and Cyclophosphamide: Preliminary Results from National Surgical Adjuvant Breast and Bowel Project Protocol B-27," Journal of Clinical Oncology, Vol. 21, No. 22, 2003, pp. 555-558. doi:10.1200/JCO.2003.12.005

[8] P. Fobair, S. L. Stewart, S. Chang, C. D’Onofrio, P. J. Banks and J. R. Bloom, "Body Image and Sexual Problems in Young Women with Breast Cancer," Psychooncology, Vol. 15, No. 7, 2006, pp. 579-594. doi:10.1002/pon.991

[9] M. Y. Nahabedian, "Breast Reconstruction: A Review and Rationale for Patient Selection," Plastic and Reconstructive Surgery, Vol. 124, No. 1, 2009, pp. 55-62. doi:10.1097/PRS.0b013e31818b8c23

[10] J. Peppercorn, "Breast Cancer in Women under 40," Oncology, Vol. 23, No. 6, 2009, pp. 465-474.

[11] J. Y. Petit, M. Rietjens and C. Garusi, "Breast Reconstructive Techniques in Cancer Patients: Which Ones, When to Apply, Which Immediate and Long Term Risks?” Journal of Hematology \& Oncology, Vol. 38, No. 3, 2001, pp. 231-239.

[12] S. J. Kronovitz and G. L. Robb, “Controversies Regarding Immediate Reconstruction: Aesthetic Risks of Radiation,” In: S. L. Spear, Ed., Surgery of the Breast, 2nd Edition, Lippincott Williams \& Wilkins, Philadelphia, 2006, pp. 679-699.

[13] S. Al Benna, "Female Plastic and Reconstructive Surgeons' Personal Decision Making for Breast Cancer Treatment and Reconstruction," Archives of Gynecology and Obstetrics, Vol. 284, No. 3, 2011, pp. 737-741. doi:10.1007/s00404-010-1721-9

[14] A. K. Alderman, S. T. Hawley, J. Waljee, M. Mujahid, M. Morrow and S. J. Katz, "Understanding the Impact of Breast Reconstruction on the Surgical Decision-Making Process for Breast Cancer," Cancer, Vol. 112, No. 3, 2008, pp. 489-494. doi:10.1002/cncr.23214

[15] M. Marín-Gutzke and A. Sánchez-Olaso, "Reconstructive Surgery in Young Women with Breast Cancer," Breast Cancer Research and Treatment, Vol. 123, Suppl. 1, 2010, pp. 67-74. 